\title{
Unexpected room-temperature ferromagnetism in bulk $\mathrm{ZnO}$
}

\author{
Yu-Chun Chen, ${ }^{1, a)}$ Eberhard Goering, ${ }^{1, b)}$ Lars Jeurgens, ${ }^{2}$ Zumin Wang, ${ }^{3}$ Fritz Phillipp, ${ }^{4}$ \\ Johannes Baier, ${ }^{5}$ Thomas Tietze, ${ }^{1}$ and Gisela Schütz ${ }^{1}$ \\ ${ }^{1}$ Department of Modern Magnetic Systems, Max-Planck-Institut für Intelligente Systeme Heisenbergstraße 3, \\ 70569 Stuttgart, Germany \\ ${ }^{2}$ Empa, Swiss Federal Laboratories for Materials Science and Technology, Überlandstraße 129, \\ CH-8600 Dübendorf, Switzerland \\ ${ }^{3}$ Department of Phase transformations, Thermodynamics and Kinetics, Max-Planck-Institut für \\ Intelligente Systeme, Heisenbergstraße 3, 70569 Stuttgart, Germany \\ ${ }^{4}$ Stuttgart Center for Electron Microscopy, Max-Planck-Institut für Intelligente Systeme Heisenbergstraße 3 , \\ 70569 Stuttgart, Germany \\ ${ }^{5}$ Institut für Materialwissenschaft Universität Stuttgart, Heisenbergstraße 3, 70569 Stuttgart, Germany
}

(Received 15 May 2013; accepted 29 September 2013; published online 16 October 2013)

\begin{abstract}
It is demonstrated that a transition from paramagnetic behavior to clear room-temperature ferromagnetism (RTFM) exists in pure bulk ZnO. A significant enhancement of RTFM has been observed in argon-annealed $\mathrm{ZnO}$ samples. Quantitative chemical analysis unambiguously indicates that oxygen-related vacancies at surface play a crucial role in this observed RTFM. We suppose that the surface magnetic states, paramagnetic in the pure nanoparticles, are converted to ferromagnetic phase after mechanical compaction. Additionally, it is found that weakly adsorbed carbon species could block the exchange coupling between isolated magnetic moments in the surface layers. (C) 2013 AIP Publishing LLC. [http://dx.doi.org/10.1063/1.4825268]
\end{abstract}

Over the past decade, $\mathrm{ZnO}$ has attracted considerable interests in the scientific community, particularly owing to its exceptional physical features such as direct wide bandgap $(\sim 3.37 \mathrm{eV})$, large exciton binding energy $(60 \mathrm{meV}$ at room temperature) and the so-called $\mathrm{d}^{0}$ ferromagnetism. ${ }^{1}$ According to theoretical predictions, ${ }^{2} \mathrm{ZnO}$ can be a potential host candidate for dilute magnetic semiconductors, which are interesting for application in spintronics. However, several experimental results have claimed that the transition-metal (TM) atoms in TM-doped $\mathrm{ZnO}$ thin film exhibit only paramagnetism, while their specimens indeed show room-temperature ferromagnetism (RTFM). ${ }^{3,4}$ In addition, an experimental report was published, presenting the existence of intrinsic ferromagnetism in pure $\mathrm{ZnO}^{5}$ However, this new magnetism is still questionable due to bad reproducibility and small magnetic moments (typically $10^{-4} \sim 10^{-3} \mathrm{emu} / \mathrm{g}$ ). Recently, more experimental evidences have reconfirmed the presence of ferromagnetism (FM) in pure $\mathrm{ZnO}$ and excluded the possible experimental error from unwanted impurities. ${ }^{6-15}$ This surprising phenomenon takes the challenge to our classical understanding of ferromagnetism, because it is thought that the ferromagnetism, especially RTFM, requires strong interacting partially filled $3 \mathrm{~d}$ and $4 \mathrm{f}$ electron shells.

In previous studies, $\mathrm{d}^{0}$ ferromagnetism was observed only in $\mathrm{ZnO}$ thin films and nanostructures ( $\leqq 100 \mathrm{~nm})$. Bulk $\mathrm{ZnO}$ is principally considered as a non-ferromagnetic material. ${ }^{16}$ Interestingly, the nature of $\mathrm{FM}$ in $\mathrm{ZnO}$ strongly depends on preparation parameters such as synthesis temperature, type of substrate, film thickness, or grain size. ${ }^{7,17}$ Probably, this is the reason why the controversy over findings from different groups leads to a confusing picture.

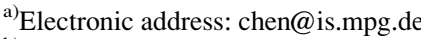

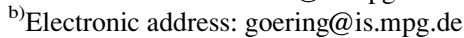

The origin of RTFM in pure $\mathrm{ZnO}$ or other nominal $\mathrm{d}^{0}$ oxides is unclear and still under debate. An acceptable assumption introduces the existence of surface magnetic moments from partially filled electron shells due to nonstochiometry and/or imperfections. Therefore, the observed ferromagnetism is induced because of strong magnetic interactions between those isolated magnetic moments. For this reason, possible origins for RTFM include all point defects, such as oxygen vacancies, ${ }^{6,10}$ oxygen interstitials, ${ }^{14}$ zinc vacancies, ${ }^{16,18}$ zinc interstitial, ${ }^{19}$ and $\mathrm{Zn}$ nanoclusters. ${ }^{20}$ Nevertheless, it remains an experimental challenge to determine which type of defects is actually responsible for RTFM. Because such defects are also a source for conducting electrons, similar arguments occur in the research on optical properties of $\mathrm{ZnO} .{ }^{12,21}$ Nevertheless, all reports claim that this RTFM is related to defects at the surface or interfaces, as proposed by the grain-boundary model. ${ }^{22}$ The idea of this model mentioned here is that paramagnetic moments are always present within grain boundaries, which can (if they participate in exchange interaction) result in ferromagnetic ordering in polycrystalline $\mathrm{ZnO}$ films.

To investigate the intrinsic magnetic properties of $\mathrm{ZnO}$, pure $\mathrm{ZnO}$ nanoparticles (purity: 99.999\%, Sigma Aldrich) was processed into pellets (4 mm diameter and $1-2 \mathrm{~mm}$ thick) under a force of $32 \mathrm{kN}$ (ca. $0.6 \mathrm{GPa}$ ) using hydraulic press. In order to avoid the introduction of extrinsic magnetic contamination, the nanoparticles were filled into clean selfdesigned Teflon molds and punches before compaction by a hardened steel mandrel. All nonmagnetic tools such as Teflon tweezers and quartz capsules, used throughout the measurements, were always cleaned before use with strong sulfuric acid solution and then distilled water. To modify the defect structure of the $\mathrm{ZnO}$ specimens, parts of the nanoparticles and also some as-prepared pellets were sealed in preevacuated quartz tube with an argon atmosphere (300 Torr, 

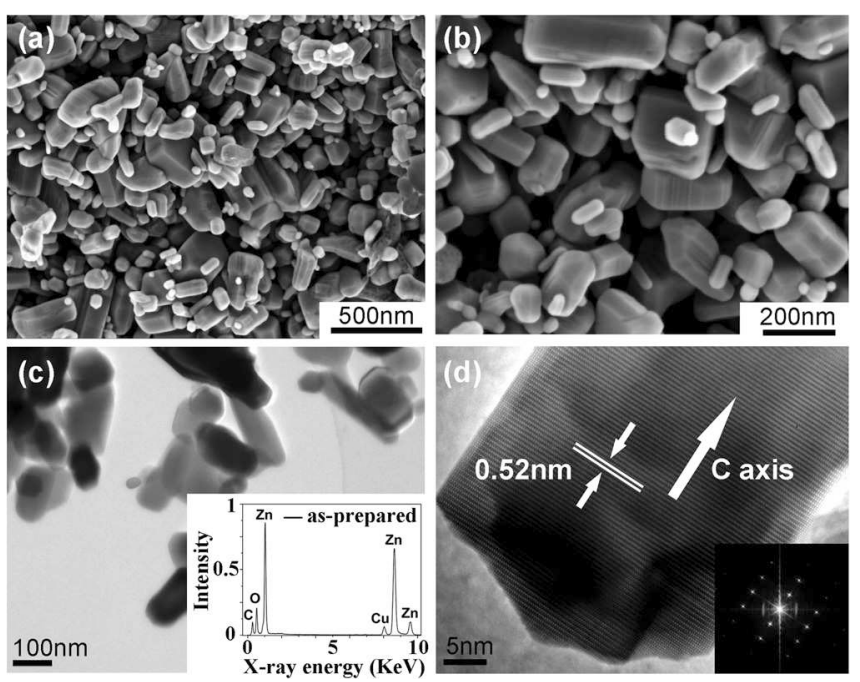

FIG. 1. SEM and TEM images of pure $\mathrm{ZnO}$ nanoparticles collected from the surface of as-prepared $\mathrm{ZnO}$ pellet. (a) Low-magnification, (b) highmagnification SEM images; (c) bright-field TEM image and EDX spectra (inset). The signals of $\mathrm{C}$ and $\mathrm{Cu}$ in EDX analysis come from copper TEM grids; (d) HRTEM image and FFT pattern (inset) indicate that the particles are elongated along the $\mathrm{c}$-axis direction.

purity: $99.999 \%)$. The sealed ampoules were heated in a conventional furnace from room temperature to $500{ }^{\circ} \mathrm{C}$ at a rate of $15^{\circ} \mathrm{C} / \mathrm{min}$, held at $500^{\circ} \mathrm{C}$ for $2 \mathrm{~h}$, and then slowly cooled down to room temperature. Finally, Ar-annealed $\mathrm{ZnO}$ nanoparticles also were shaped into pellets in the same way as described above but with three different forces of 30, 32, and $34 \mathrm{kN}$.

The microstructure and morphology of the $\mathrm{ZnO}$ pellets were investigated by scanning electron microscopy (SEM) and transmission electron microscopy (TEM) shown in Fig. 1. The size distribution of the $\mathrm{ZnO}$ nanoparticles is quite broad, approximately ranging from 30 to $300 \mathrm{~nm}$ as displayed in Fig. 1(b). Figure 1(c) presents a low magnification TEM bright-field image of the nanoparticles collected from the top surface of one as-prepared pellet. The inset exhibits their chemical composition by Energy dispersive spectroscopy (EDX), which excludes the presence of magnetic impurities within the instrumental detection limit. As observed by the high-resolution TEM (HRTEM) image and the corresponding FFT pattern, the $\mathrm{ZnO}$ nanoparticles are principally single-crystalline with a hexagonal lattice in Fig. 1(d). The lattice spacing of $0.52 \mathrm{~nm}$, as determined by HRTEM analysis, corresponds to a d-spacing of [001] crystal planes that the nanoparticles are elongated along the c-axis direction. Nearly, the same nanostructures are found in Arannealed samples; hence, only the images from as-prepared pellets are presented here.

Figure 2(a) displays the X-ray diffraction (XRD) patterns of three different samples for phase identification. All observed peaks can be indexed to the Wurtzite phase of $\mathrm{ZnO}$ (Space group $\mathrm{P} 6_{3} \mathrm{mc}$; lattice parameters $a=3.2498 \AA$ and $\mathrm{c}=5.2066 \AA$ ), consistent with the values in the standard card (JCPDS card No. 36-1451). No other phases due to impurities or residues were detected within the experimental resolution. Moreover, the Ar-annealed pellet does not show significant change in size-dependent peak width. Since all of the reported FM signals in pure $\mathrm{d}^{0}$ oxides show very low (a)

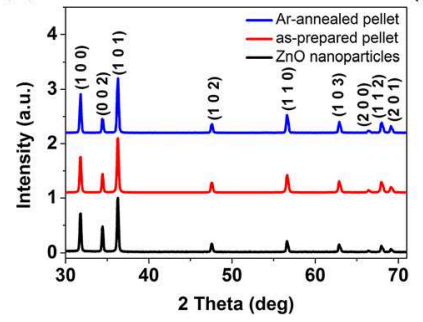

(b)

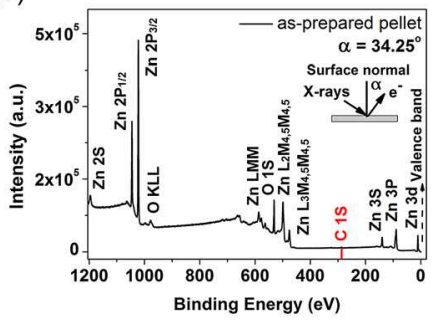

FIG. 2. (a) $\mathrm{X}$-ray diffraction patterns of pure $\mathrm{ZnO}$ nanoparticles, as-prepared $\mathrm{ZnO}$ pellet, and Ar-annealed $\mathrm{ZnO}$ pellet. All diffraction peaks can be indexed to a Wurzite phase of $\mathrm{ZnO}$. No apparent change in structure is induced after Ar-annealing. (b) XPS survey spectrum of as-prepared pellet (red bar corresponds to $\mathrm{C} 1 \mathrm{~s}$ peak).

magnetic signal, the nominal chemical composition of our $\mathrm{ZnO}$ specimens was also examined by inductively coupled plasma optical emission spectroscopy (ICP-OES). The analytical results (listed in Table I) indicate that the concentration of trace magnetic elements, such as iron, is below the instrument detection limit. If we assume that some magnetic ions are present in the samples at the level of ICP-OES detection limit, they still cannot account for the obtained FM signals presented below. ${ }^{13,23}$

The chemical constitution at the outermost surface of the $\mathrm{ZnO}$ pellets was further characterized by angle-resolved X-ray photoelectron spectroscopy (AR-XPS). All XPS spectra were obtained in the 'parallel detection and standard lens mode' simultaneously collecting all photoelectrons over the detection angle range between $23^{\circ}$ and $83^{\circ}$ (step size $=0.2 \mathrm{eV}$; pass energy $=200 \mathrm{eV}$; energy range $=0-1200 \mathrm{eV}) .{ }^{24}$ Only $\mathrm{Zn}$ and $\mathrm{O}$ related photo- and Auger-electrons were detected in XPS profiles, and even adventitious carbon is negligible (see Fig. 2(b)). This implies that the samples in this work are extremely pure, and the unwanted contribution from extrinsic pollution can be ignored.

For studying the magnetic properties, all of the specimens were fixed tightly by clean self-designed quartz capsules when the samples were measured in the superconducting quantum interference device magnetometer (SQUID). The $\mathrm{M}(\mathrm{H})$ loops of quartz capsules were recorded separately in advance, as required for "background subtraction." Detailed analysis of the measured data ensures that background signal does not obscure the phenomenon of interest (see supplementary material ${ }^{32}$ ). Also, no apparent signal change was found from the used capsule after cleaning with strong sulfuric acid solution. It is noted that the background signal was removed from all shown SQUID data (raw and processed curves), and the process of data treatment is described in supplemental material. ${ }^{32}$ Besides, pure $\mathrm{ZnO}$ nanoparticles were measured as a reference, which exhibit only diamagnetism superimposed with a weak paramagnetic component over the temperature range of 5-300 K (Fig. 3(a)). Similarly, the raw $\mathrm{M}(\mathrm{H})$ curves of as-prepared $\mathrm{ZnO}$ pellets also present diamagnetic

TABLE I. Concentration of each trace element determined by ICP-OES.

\begin{tabular}{lccccccc}
\hline \hline Element [ppm] & $\mathrm{Cr}$ & $\mathrm{Cu}$ & $\mathrm{Fe}$ & $\mathrm{Mg}$ & $\mathrm{Mn}$ & $\mathrm{Ni}$ & $\mathrm{Co}$ \\
\hline ZnO nanoparticles & $<0.5$ & $<1.0$ & $<2.0$ & $<1.0$ & $<0.5$ & $<2.0$ & $<1.0$ \\
as-prepared ZnO pellet & $<0.5$ & $<1.0$ & $<2.0$ & $<1.0$ & $<0.5$ & $<2.0$ & $<1.0$ \\
\hline \hline
\end{tabular}


(a)

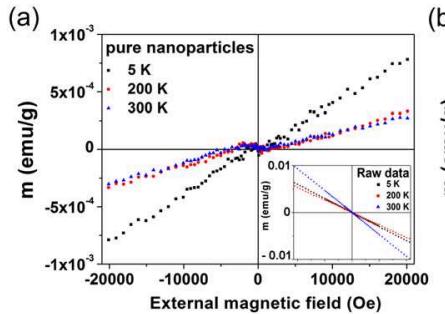

(c)

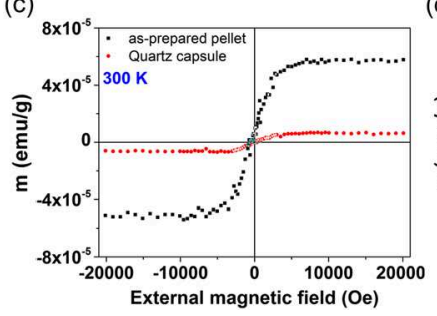

(b)

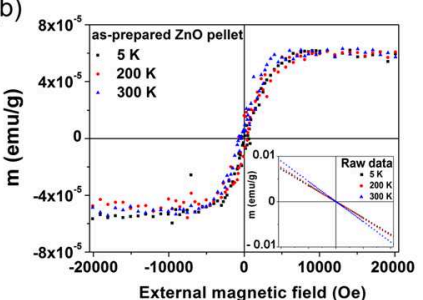

(d)

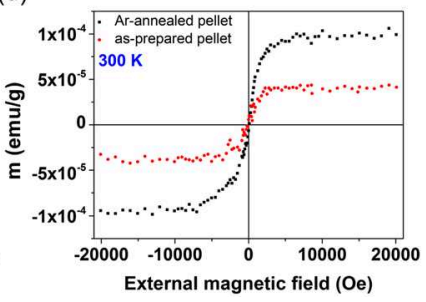

FIG. 3. (a) $\mathrm{M}(\mathrm{H})$ curves from pure $\mathrm{ZnO}$ nanoparticles after subtracting diamagnetic contribution. (b) $\mathrm{M}(\mathrm{H})$ curves from as-prepared $\mathrm{ZnO}$ pellet present typical ferromagnetic behavior after subtracting diamagnetic contribution. The insets in (a) and (b) show the corresponding raw data, indicating diamagnetic contribution is the dominant component. (c) Comparison between as-prepared $\mathrm{ZnO}$ pellet and quartz capsule used for SQUID measurements. (d) RTFM curve from Ar-annealed pellet compared to as-prepared one.

signal predominantly at various temperatures (the inset in Fig. 3(b)). The average diamagnetic susceptibility in asprepared pellets of $\sim-2.45 \times 10^{-7} \mathrm{emu} / \mathrm{gOe}$ is in good agreement with other reported values measured from $\mathrm{ZnO}$ nanoparticles. ${ }^{8,25}$ To compare the pellets used in this study with bulk samples, the magnetic properties of a pure $\mathrm{ZnO}$ single crystal (MaTeck $\mathrm{GmbH}$ ) were examined, giving a diamagnetic susceptibility of about $-3.7 \times 10^{-7} \mathrm{emu} / \mathrm{gOe}$. Such a clear difference with respect to the pressed pellets indicates that the RT paramagnetic susceptibility of $\mathrm{ZnO}$ pellets is about $>1.25 \times 10^{-7} \mathrm{emu} / \mathrm{gOe}$, which is almost two orders of magnitude larger than the estimated upper limit of magnetic signals from possible impurity concentrations determined by ICP-OES measurement (see above). Therefore, we can conclude that the observed paramagnetism in nanoparticles and as-prepared pellets is not due to extrinsic contaminations. The most striking result found in this work is that RTFM could be simply switched on just by pressing $\mathrm{ZnO}$ nanoparticles into pellets. Figure 3(b) shows the clear ferromagneticlike loops from as-prepared pellets after subtracting their own diamagnetic contribution. The saturation magnetization (Ms) of the recorded ferromagnetic-like loops is $\sim 5.5 \times 10^{-5}$ $( \pm 0.5) \mathrm{emu} / \mathrm{g}$ with a coercivity of about $350( \pm 50) \mathrm{Oe}$. We note that the FM of pellets seems to be temperatureindependent over the scan temperature range, suggesting the Curie temperature is far above room temperature. Figure 3(c) presents the direct comparison of $\mathrm{M}(\mathrm{H})$ curves from as-prepared pellet and quartz capsule, demonstrating that the measured RTFM in $\mathrm{ZnO}$ pellets does not originate from spurious background effect. It is worth to note that the ferromagnetic state in $\mathrm{ZnO}$ pellets is highly reproducible.

It has been assumed that oxygen vacancies are possible candidates for RTFM in oxides and can be simply removed or introduced into $\mathrm{ZnO}$ by annealing in air or argon, respectively. In order to verify this hypothesis, as-prepared pellets were post-annealed in an argon atmosphere at $500^{\circ} \mathrm{C}$ for 2 h. In Fig. 3(d), it is clear that the RTFM of Ar-treated

pellets is enhanced prominently in agreement with Refs. 12 and 26.

To elucidate the relationship between argon treatment and the RTFM of $\mathrm{ZnO}$, the $\mathrm{O} 1 \mathrm{~s}$ and $\mathrm{Zn} \mathrm{2p3/2-1/2} \mathrm{spin} \mathrm{orbit}$ doublet spectra were recorded at near-normal (more bulk sensitive) and grazing (more surface sensitive) detection angles of $\alpha=34.25^{\circ}$ and $\alpha=71.75^{\circ}$, respectively. Spectral reconstruction of each region was performed by linear-least-squares fitting after subtraction of a Shirley-type inelastic background. In the $\mathrm{O} 1 \mathrm{~s}$ region, there are two different distinct peaks (see Figs. 4(a) and 4(b)). The main O $1 \mathrm{~s}$ peak at the lower binding energy (LBE, $530.8 \mathrm{eV}$ ) side of the fitting envelop originates from lattice oxygen anions $\left(\mathrm{O}^{2-}\right)$ in bulk $\mathrm{ZnO}$, whereas the $\mathrm{O} 1 \mathrm{~s}$ peak with higher-binding energy (HBE, 532.4 eV) is typically attributed to surface hydroxide $\operatorname{groups}^{27}$ or oxygen-deficient regions. ${ }^{10}$ Indeed, the effective depths analysis confirms that the resolved HBE O $1 \mathrm{~s}$ peak refers to chemical species at the oxide surface. ${ }^{28}$ Fourier transform infrared (FTIR) spectra indicate that the HBE O $1 \mathrm{~s}$ peak must be related to surface $\mathrm{OH}$ groups in terms of the change in $\mathrm{O}-\mathrm{H}$ stretching modes (see supplemental material ${ }^{32}$ for more detail). In addition, it is found that the RTFM signal decreases or even disappears if a certain amount of carbon-related species has adsorbed on sample surface (results not shown here). Therefore, we suggest that carbon-free surface is necessary to study this surprising FM feature.

In addition to identification of surface chemical composition, the average atom ratio $\frac{C_{Z n}}{C_{O}}$ could be estimated quantitatively from the resolved intensities of the $\mathrm{Zn} 2 \mathrm{p} 3 / 2$ main peak $\left(I_{Z n 2 p 3 / 2}\right)$ and the $\mathrm{O} 1 \mathrm{~s}$ peak $\left(I_{O 1 s}\right.$; the sum area of the LBE and HBE O $1 \mathrm{~s}$ peaks) at each detection angle, according to ${ }^{24}$

$$
\begin{aligned}
\frac{C_{Z n}}{C_{O}}= & \frac{I_{Z n 2 p 3 / 2}}{I_{O 1 s}} \times \frac{\lambda_{O 1 s}}{\lambda_{Z n 2 p 3 / 2}} \times \frac{\sigma_{O 1 s}}{\sigma_{Z n 2 p 3 / 2}} \times \frac{T X F N_{O 1 s}}{T X F N_{Z n 2 p 3 / 2}} \\
& \times \frac{W_{O 1 s}}{W_{Z n 2 p 3 / 2}},
\end{aligned}
$$

(a)

(c)

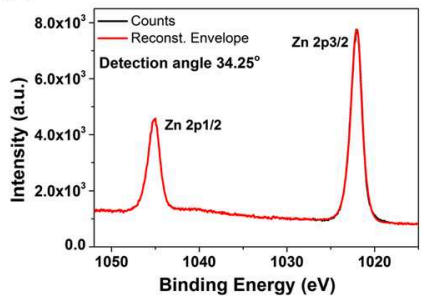

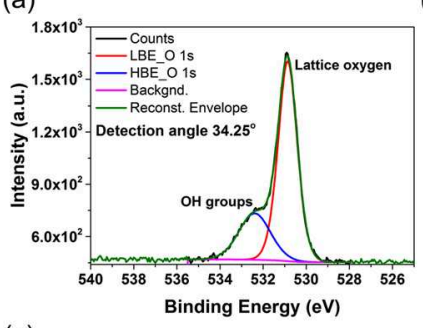

(b)

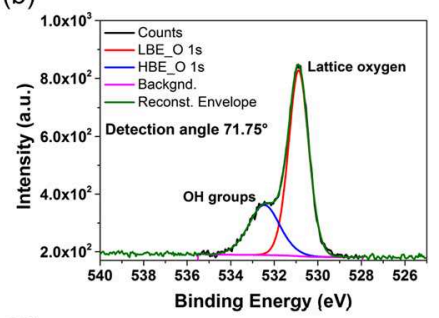

(d)

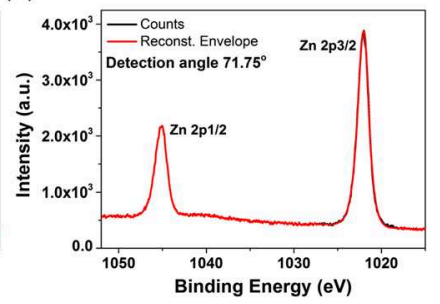

FIG. 4. Reconstruction of the (a) and (b) O $1 \mathrm{~s}$ and (c) and (d) Zn 2p3/2-1/2 peaks from the as-prepared $\mathrm{ZnO}$ pellets recorded at a bulk- and surfacesensitive detection angle of (a) and (c) $34.25^{\circ}$ and (b) and (d) $71.75^{\circ}$, respectively. 
TABLE II. Average $\mathrm{Zn} / \mathrm{O}$ atom ratio and the relative intensity of the resolved HBE O $1 \mathrm{~s}$ peak (as compared to the sum of the LBE and HBE O $1 \mathrm{~s}$ intensities) at bulk- and surface-sensitive detection angles of $34.25^{\circ}$ and $71.75^{\circ}$.

\begin{tabular}{lccc}
\hline \hline Specimen & $\mathrm{Zn} / \mathrm{O}$ & $\mathrm{OH}\left(34.25^{\circ}\right)[\%]$ & $\mathrm{OH}\left(71.75^{\circ}\right)[\%]$ \\
\hline As-prepared ZnO pellet & 1.23 & 27.4 & 29.5 \\
Ar-annealed ZnO pellet & 1.25 & 25.5 & 28.2 \\
\hline \hline
\end{tabular}

where $\sigma_{O 1 s}$ and $\sigma_{Z n 2 p 3 / 2}$ are the total photoionization crosssections of the corresponding photoelectron lines; $T X F N_{O 1 s}$ and $T X F N_{Z n 2 p 3 / 2}$ are the analyzer transmission functions; $\lambda_{O 1 s}$ and $\lambda_{Z n 2 p 3 / 2}$ are the inelastic mean free paths of the detected $\mathrm{O} 1 \mathrm{~s}$ and $\mathrm{Zn} 2 \mathrm{p} 3 / 2$ photoelectrons; and $W_{O 1 s}$ and $W_{Z n 2 p 3 / 2}$ are the anisotropies of the photoionization cross-sections. The determined average $\mathrm{Zn} / \mathrm{O}$ ratio in the as-prepared $\mathrm{ZnO}$ pellet is 1.23 at detection angle $\alpha=34.25^{\circ}$, which means that the as-prepared pellets are substoichiometric $\left(\mathrm{ZnO}_{1-x}, 0<x<1\right)$ on average. This result agrees well with previous reports, ${ }^{29,30}$ which claim that $\mathrm{ZnO}$ is an intrinsic semiconductor due to intrinsic oxygen vacancies. The evaluated $\mathrm{Zn} / \mathrm{O}$ ratio in the Ar-annealed pellets shows that the degree of substoichiometry is slightly enhanced after the post-thermal treatment; see Table II. It was found that zinc atoms basically do not escape when the heating temperature is below $500{ }^{\circ} \mathrm{C}$; hence, the thermal treatment in this study could mainly promote the formation of oxygen-related vacancies, in accordance with Ref. 31.

The increase in the average $\mathrm{Zn} / \mathrm{O}$ ratio suggests that there is potential relationship between RTFM and oxygen-related vacancies, which are probably associated with the release of surface $\mathrm{OH}$ groups during Ar-annealing as discovered in the corresponding FTIR spectra. Figure 5(a) shows that the magnetization of the $\mathrm{ZnO}$ samples (pre_30 kN, pre_32 $\mathrm{kN}$ and pre_34 $\mathrm{kN}$ ) could be much further enhanced if the pellets are made from Ar-annealed nanoparticles and pressed under increasing forces $(30,32$, and $34 \mathrm{kN})$. No clear difference was found between the XPS analysis of Ar-annealed pellet and Ar-annealed nanoparticles (Fig. 5(b)). Therefore, the (a)

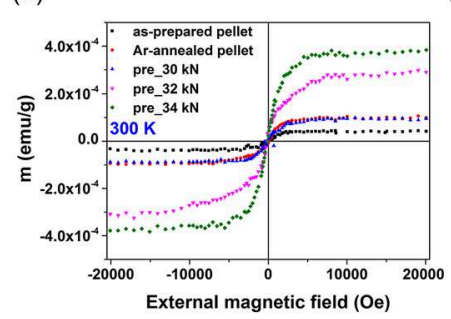

(c)

c)

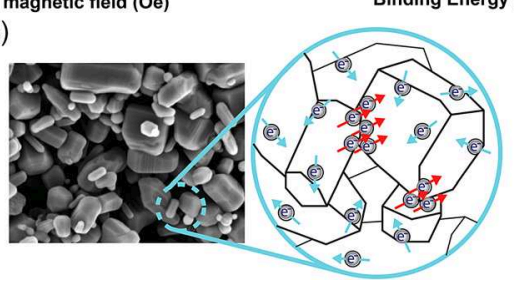

(b)

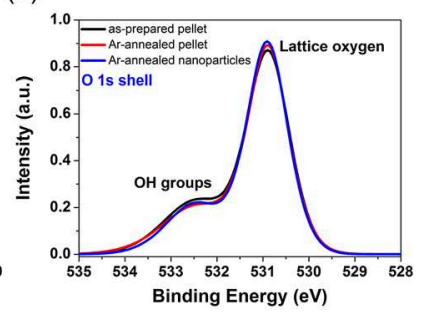

FIG. 5. (a) RTFM curves from as-prepared and Ar-treated $\mathrm{ZnO}$ pellet. (b) XPS analysis on O $1 \mathrm{~s}$ peak before and after Ar-annealing. Each profile is normalized to LBE O $1 \mathrm{~s}$ area with energy scale referenced to $\mathrm{Zn} 2 \mathrm{p} 3 / 2$ peak. (c) Schematic illustration of ferromagnetic exchange coupling in the bulk pellets. increased amount of oxygen-related vacancies is the potential reason to find stronger RTFM in the pellets which are made from Ar-annealed nanoparticles. For a given surface, raising compaction force results in higher compaction density, which means individual particles have more contact with their neighbors, leading to long range interaction. Therefore, a systematic effect on magnetization was evidently observed as a function of pressing force. However, the exact bulk density of these specimens is unknown and requires further examination.

As illustrated in Fig. 5(c), a possible explanation for this experimental finding is that sufficient isolated magnetic moments due to surface oxygen-related vacancies can participate in ferromagnetic exchange coupling after mechanical compression. These magnetic moments should be essentially present because nanoparticles with high surface-to-volume ratio have a higher density of surface oxygen-related vacancies. This assumption is strongly supported by the observed paramagnetism in pure nanoparticles. The enhanced RTFM signals in Ar-annealed pellets also indicate that oxygenrelated vacancies play a crucial role. In addition, the effect of Ar-annealing is either to create oxygen-related vacancies or remove adventitious carbon species. Therefore, we propose that adsorbed carbon species can interfere, to some extent, with the coupling between surface magnetic moments. Without detrimental effect from adventitious carbon (almost inevitable in practice), we show here that RTFM can be also found in bulk $\mathrm{ZnO}$ having relatively larger grain size as compared to the threshold value proposed from grainboundary model. This is maybe the reason why RTFM is difficult to be highly reproducible in some reports.

In conclusion, we have reported that ferromagnetism can be induced in bulk $\mathrm{ZnO}$ by pressing dia/paramagnetic nanoparticles into pellets. The observed RTFM can be explained by the interaction between isolated spin moments existing in surface oxygen-related vacancies, which could be associated with HBE O $1 \mathrm{~s}$ peak as determined by AR-XPS. Furthermore, we suggest that the clean surface structure seems an important requirement to observe $\mathrm{RTFM}$ in $\mathrm{ZnO}$. The actual effect of weakly adsorbed carbon-related species on RTFM needs more investigation in future. Nevertheless, this work provides clear evidences that RTFM can be induced in pure bulk $\mathrm{ZnO}$. It is believed that the magnitude of ferromagnetic signal can be further improved with much higher compression pressure and optimum thermal treatment. Because the annealed samples are quite rigid and remain intact in the ultrasonic bath (water or ethanol) unlike as-prepared pellets, the sample preparation in this work maybe provides another route for the research of ferromagnetism in TM-doped oxides.

We would like to thank Dr. Andreas Leineweber and Gerd Maier for XRD measurement. The electron microscopy, FTIR and XPS operation were supported by Peter Kopold, Wolfgang König, and Michaela Wieland, respectively. Bernd Ludescher, Thomas Meisner, and Arnold Weible provided valuable advice for sample preparation and post-thermal treatment.

\footnotetext{
${ }^{1}$ J. M. D. Coey, Solid State Sci. 7, 660 (2005).

${ }^{2}$ T. Dietl, H. Ohno, F. Matsukura, J. Cibert, and D. Ferrand, Science 287, 1019 (2000).
} 
${ }^{3}$ M. Gacic, G. Jakob, C. Herbort, H. Adrian, T. Tietze, S. Brück, and E. Goering, Phys. Rev. B 75, 205206 (2007).

${ }^{4}$ T. S. Herng, D.-C. Qi, T. Berlijn, J. B. Yi, K. S. Yang, Y. Dai, Y. P. Feng, I. Santoso, C. Sanchez-Hanke, X. Y. Gao, A. T. S. Wee, W. Ku, J. Ding, and A. Rusydi, Phys. Rev. Lett. 105, 207201 (2010).

${ }^{5}$ A. Sundaresan, R. Bhargavi, N. Rangarajan, U. Siddesh, and C. N. R. Rao, Phys. Rev. B 74, 161306 (2006).

${ }^{6}$ D. Gao, Z. Zhang, J. Fu, Y. Xu, and J. Qi, J. Appl. Phys. 105, 113928 (2009).

${ }^{7}$ R. Podila, W. Queen, A. Nath, J. T. Arantes, A. L. Schoenhalz, A. Fazzio, G. M. Dalpian, J. He, S. J. Hwu, M. J. Skove, and A. M. Rao, Nano Lett. 10, 1383 (2010).

${ }^{8}$ Q. Xu, S. Zhou, and H. J. Schmidt, J. Alloys Compd. 487, 665 (2009).

${ }^{9}$ R. Escudero and R. Escamilla, Solid State Commun. 151, 97 (2011).

${ }^{10}$ W. Liu, W. Li, Z. Hu, Z. Tang, and X. Tang, J. Appl. Phys. 110, 013901 (2011).

${ }^{11}$ X. Y. Xu, C. X. Xu, J. Dai, J. G. Hu, F. J. Li, and S. Zhang, J. Phys. Chem. C 116, 8813 (2012).

${ }^{12}$ P. Zhan, W. Wang, C. Liu, Y. Hu, and Z. Li, J. Appl. Phys. 111, 033501 (2012).

${ }^{13}$ J. I. Hong, J. Choi, S. S. Jang, J. Gu, Y. Chang, G. Wortman, R. L. Snyder, and Z. L. Wang, Nano Lett. 12, 576 (2012).

${ }^{14}$ H. You, J. Yang, J. Y. Zhu, W. F. Xu, and X. D. Tang, Appl. Surf. Sci. 258, 4455 (2012).

${ }^{15}$ S. Banerjee, M. Mandal, N. Gayathri, and M. Sardar, Appl. Phys. Lett. 91, 182501 (2007).

${ }^{16}$ N. H. Hong, J. Sakai, and V. Brizé, J. Phys.: Condens. Matter 19, 036219 (2007).

${ }^{17}$ G. Z. Xing, Y. H. Lu, Y. F. Tian, J. B. Yi, C. C. Lim, Y. F. Li, G. P. Li, D. D. Wang, B. Yao, J. Ding, Y. P. Feng, and T. Wu, AIP Adv. 1, 022152 (2011).
${ }^{18}$ M. Khalid, M. Ziese, A. Setzer, P. Esquinazi, M. Lorenz, H. Hochmuth, M. Grundmann, D. Spemann, T. Butz, G. Brauer, W. Anwand, G. Fischer, W. A. Adeagbo, W. Hergert, and A. Ernst, Phys. Rev. B 80, 035331 (2009).

${ }^{19}$ X. Zhang, Y. H. Cheng, L. Y. Li, H. Liu, X. Zuo, G. H. Wen, L. Li, R. K. Zheng, and S. P. Ringer, Phys. Rev. B 80, 174427 (2009).

${ }^{20}$ J. B. Yi, H. Pan, J. Y. Lin, J. Ding, Y. P. Feng, S. Thongmee, T. Liu, H. Gong, and L. Wang, Adv. Mater. 20, 1170 (2008).

${ }^{21}$ Y. W. Heo, D. P. Norton, and S. J. Pearton, J. Appl. Phys. 98, 073502 (2005).

${ }^{22}$ B. Straumal, A. Mazilkin, S. Protasova, A. Myatiev, P. Straumal, G. Schütz, P. Aken, E. Goering, and B. Baretzky, Phys. Rev. B 79, 205206 (2009).

${ }^{23}$ R. Salzer, D. Spemann, P. Esquinazi, R. Höhne, A. Setzer, K. Schindler, H. Schmidt, and T. Butz, J. Magn. Magn. Mater. 317, 53 (2007).

${ }^{24}$ L. P. H. Jeurgens and M. S. Vinodh, Surf. Interface Anal. 36, 1629 (2004).

${ }^{25}$ S. Zhou, K. Potzger, H. Reuther, K. Kuepper, W. Skorupa, M. Helm, and J. Fassbender, J. Appl. Phys. 101, 09H109 (2007).

${ }^{26}$ D. Sanyal, M. Chakrabarti, T. K. Roy, and A. Chakrabarti, Phys. Lett. A 371, 482 (2007).

${ }^{27}$ T. Li, C. S. Ong, T. S. Herng, J. B. Yi, N. N. Bao, J. M. Xue, Y. P. Feng, and J. Ding, Appl. Phys. Lett. 98, 152505 (2011).

${ }^{28}$ L. P. H. Jeurgens, M. S. Vinodh, and E. J. Mittemeijer, Appl. Surf. Sci. 253, 627 (2006).

${ }^{29}$ L. Q. Jing, F. L. Yuan, H. G. Hou, B. F. Xin, W. M. Cai, and H. G. Fu, Sci. China. Ser. B-Chem. 48, 25 (2005).

${ }^{30}$ J. Singh, A. Srivastava, R. S. Tiwari, and O. N. Srivastava, J. Nanosci. Nanotechnol. 5, 2093 (2005).

${ }^{31}$ S. Dutta, M. Chakrabarti, S. Chattopadhyay, and D. Janab, J. Appl. Phys. 98, 053513 (2005).

${ }^{32}$ See supplementary material at http://dx.doi.org/10.1063/1.4825268 more information about data analysis and FTIR spectra. 